\title{
Mitteilungen
}

\section{Kollegium für Hausarztmedizin KHM}

\section{KHM Forschungspreis Hausarzt- medizin 2012}

Fachgebiet: Hausarztmedizin

Arbeiten/Kriterien: Abgeschlossene wissenschaftliche Arbeiten aus der Schweiz oder von im Ausland tätigen Schweizerinnen und Schweizern, die wichtige Aspekte hausärztlicher Grundversorgung thematisieren, insbesondere: die Qualität der Behandlung und der Betreuung hausärztlicher Patienten, die praktische Arbeit des hausärztlichen Grundversorgers (valid, relevant, umsetzbar im Rahmen der Praxis), die Sicherstellung der hausärztlichen Grundversorgung (Erforschung der Grundlagen, Arbeitsbedingungen, Ressourcenlage und Versorgungssituation).

Preissumme: 30000 Franken, gestiftet von Mepha Pharma AG. Es können eine oder mehrere Arbeiten ausgezeichnet werden. Bei der Preisvergabe an mehrere Arbeiten wird die Preissumme aufgeteilt. Der Rechtsweg ist ausgeschlossen.

Teilnehmer/innen: Autorinnen und Autoren aus der Schweiz oder von im Ausland tätigen Schweizern, die in den vergangenen drei Jahren eine bedeutende hausärztliche Arbeit abgeschlossen haben.

Eingabetermin: 1. Dezember 2011 (bitte den neuen, früheren Termin beachten!)

Preisverleihung: 21. Juni 2012 anlässlich KHMFortbildungskongress Luzern (21./22.6.2012) und am 30. August 2012 bei den Journées de formation CMPR / Swiss Family Docs Conference in Lausanne (30./31.8.2012).

Preiskomitee: eine unabhängige Jury, eingesetzt vom Stiftungsrat KHM.

Teilnahmebedingungen: Einzureichen sind in elektronischer Form per E-Mail oder CD (keine Disketten) und auf Papier: Anmeldeformular (www.kollegium.ch/rd/d.html), Curriculum vitae des Hauptautors, Manuskript, Begleitschreiben «Bedeutung der eingereichten Arbeit für die Hausarztmedizin». Schon einmal unterbreitete Arbeiten können nicht berücksichtigt werden und über die Preisnominierung wird keine Korrespondenz geführt.

Auskunft: Kollegium für Hausarztmedizin KHM, Sekretariat Forschung Hausarztmedizin, Landhausweg 26, 3007 Bern. foham@ kollegium.ch; www.kollegium.ch/rd/d.html

\section{Schweizerische Psoriasis und Vitiligo Gesellschaft (SPVG)}

\section{Neuer Psoriasis-Ratgeber}

Von allen Seiten beleuchtet wird das Thema Schuppenflechte in einem Psoriasis-Ratgeber, der von der Schweizerischen Psoriasis und Vitiligo Gesellschaft (SPVG) herausgegeben wurde.

Die SPVG ist eine Patientenorganisation für Schuppenflechte (Psoriasis) und Weissfleckenkrankheit (Vitiligo). Im Ratgeber wird erläutert, worum es sich bei Psoriasis handelt, welche Formen es gibt und wodurch diese Hautkrankheit ausgelöst werden kann. Die diversen klassischen und modernen, medizinischen und alternativen Therapieformen werden ebenso angesprochen wie die tägliche Pflege der Haut. Spezielle Themen wie Depression, Ernährung, Alkohol, Rauchen, Sexualität, Schwangerschaft oder Psoriasis bei Kindern vervollständigen die allgemeinen Informationen. Ergänzt wird der Ratgeber durch eine Liste von Regionalgruppen der SPVG und weiteren Patientenorganisationen. Allgemeine Ratschläge und weiterführende Literatur finden sich ebenfalls.

Der Ratgeber kann bezogen werden beim SPVG-Sekretariat, Scheibenstr. 20, 3014 Bern, Tel. 03135990 99, info@spvg.ch

\section{evidentia}

evidentia.ch - Informationsplattform von Ärzten für Ärzte

Das schweizerische Evidenzportal für Diagnostik und Therapie www.evidentia.ch stellt kostenlos ständig aktualisierte, evidenzbasierte Informationen für Schweizer Fachärzte in Klinik, Praxis und Ausbildung zur Verfügung. Slide Kits zum Download geben einen umfassenden Überblick über eine Indikation (z. B. Diagnose und Therapie des Hirnschlags) oder beleuchten ausgewählte Aspekte innerhalb einer Indikation (z. B. MS und Kognition). Zusätzlich stehen Hilfsmittel, Tests, Scores und Tabellen für Diagnose und Differentialdiagnose für den täglichen Bedarf in der Praxis bereit. Alle Informationen stehen auch zur Weiterverwendung (z. B. in Präsentationen) zur Verfügung. Das Konzept von evidentia beruht auf Neutralität und wissenschaftlicher Genauigkeit: Die Inhalte werden von Fachärzten und Opinion Leaders aus den jeweiligen Indikationsgebieten in einem mehrstufigen Prozess entwickelt und überprüft sowie regelmässig aktualisiert. Sponsoren unterstützen das Portal, haben jedoch keinen Einfluss auf den Inhalt.

evidentia startet mit dem Fachgebiet Neurologie. Das Portal wird nach und nach um weitere Indikations- und Fachgebiete erweitert, Ende des Jahres wird die französische Version aufgeschaltet. 